\title{
Seis hipótesis de trabajo para entender la delincuencia y el miedo al delito
}

\author{
Baltasar Fernández-Ramírez \\ Universidad de Almería \\ Laboratorio de Evaluación del Diseño Ambiental
}

\begin{abstract}
RESUMEN
La inseguridad ciudadana y el miedo al delito son uno de los tópicos de investigación más frecuentes en la literatura actual sobre estudios urbanos. La abundancia de estudios empíricos exige una reflexión teórica profunda que abra nuevas vías de trabajo e integre los resultados que parecen aceptados entre la comunidad de investigadores. En este artículo, expondremos seis hipótesis o marcos teóricos que podrían resultar relevantes para este fin. Acudo a la teoría social urbana en busca de ideas, desde los autores clásicos del siglo XIX hasta las nuevas propuestas narrativas posmodernas. La conclusión apunta hacia el mayor potencial de los marcos ecológico y narrativo.
\end{abstract}

Palabras clave. Miedo al delito. Ecología urbana. Teoría social urbana. Psicología de la ciudad.

\begin{abstract}
Fear of crime is one of the most frequent topics of research in the current literature on urban studies. The abundant empirical work calls for a deep theoretical reflection in order to open new lines of research and integrate many results well-accepted in the community of specialists. In this paper, I will describe six hypothesis or theoretical frames that could be relevant to this goal. I will look into the urban social theory searching for ideas, from the classic authors in the XIX century, till the new postmodern narrative proposals. The paper concludes emphasizing the bigger potential of the ecological and narrative theoretical frames.
\end{abstract}

Keywords. Fear of crime. Urban ecology. Urban social theory. Urban psychology. 


\section{Introducción}

Este texto es una reproducción libre de la ponencia impartida en las Jornadas de Seguridad ciudadana y Miedo al crimen en el Instituto Vasco de Criminología durante el mes de enero de 2006. Como el auditorio es ahora un lector ausente, y no siento la presión de sus miradas ni la necesidad de improvisar argumentos y ejemplos que ilustren mis ideas, aprovecharé para eliminar muchas licencias de lenguaje utilizadas en aquella ponencia, cuidar la expresión y tratar de exponer unas cuantas reflexiones personales con la claridad que me da el estado actual de mi conocimiento sobre el tema.

La preparación de la ponencia me obligó entonces a retomar unos temas que tenía algo abandonados, y hacer el esfuerzo de recuperar algunos textos del archivador, buscar otros nuevos y leer todos ellos con renovada ilusión y detenimiento. El fruto de este esfuerzo es lo que aquí trataré de exponer, con los defectos derivados de la necesidad de recomponer mi propio discurso ocho años después de mi última publicación sobre inseguridad y miedo al delito El resultado de esta recomposición no está cerrado y quizá produzca una acertada sensación de discurso inacabado. Ruego al indulgente lector y a mis ilustres colegas que sepan disculpar estas limitaciones. Sólo son ideas para seguir trabajando. Me conformaré con que inciten a la reflexión y animen a algún joven investigador a iniciar nuevos trabajos sobre el tema que nos ocupa.

Tenía varias opciones sobre cómo plantear la exposición. Con la más convencional, por ejemplo, habría repasado los modelos teóricos aceptados, los principales resultados y líneas de investigación, así como los modelos de intervención mejor conocidos. Sin embargo, a riesgo de ser criticado, he escogido un estilo de exposición más relajada, dado que me encuentro en un período personal de reflexión que me ha traído a dudar de las estructuras de discurso de la corriente principal de la psicología, incluidas las rutinas de investigación y la retórica formal de las publicaciones. Tampoco tengo datos nuevos que contar, es cierto. Sencillamente, mi objetivo será exponer unas cuantas reflexiones teóricas que ayuden a enmarcar la investigación sobre la inseguridad y el delito, sugerir nuevas líneas de trabajo y quizá criticar y reinterpretar los datos ya conocidos.

Revista Española de Investigación Criminológica

Artículo 6, Número 6 (2008) $\quad$ www.criminología.net

ISSN: $1696-9219$ 
En un primer epígrafe, plantearé la necesidad de realizar una reflexión crítica en profundidad, ante la aparente sensación de agotamiento conceptual de la investigación sobre delincuencia e inseguridad. A continuación, a modo de posibilidades, iré desgranando hasta seis hipótesis o marcos teóricos que pudieran ser relevantes para este fin. Concluiré después con brevedad, señalando el mayor potencial del marco ecológico y defendiendo el interés de una hipótesis narrativa y posmoderna, en línea con la actualidad teórica de las ciencias sociales y las nuevas propuestas del urbanismo.

\section{La situación actual}

El punto de partida que tenemos es una cantidad amplia y aceptada de resultados empíricos y propuestas teóricas de muy diversas investigaciones en torno al contexto social de la inseguridad, la dimensión ambiental del delito y las denominadas paradojas del delito (Fernández-Ramírez, 1995, 2000; Hale, 1996; San Juan, Vergara y Germán, 2005). Permítanme enumerar algunas. Aceptamos que existe una distinción entre el sentimiento difuso de inseguridad que se registra mediante las encuestas masivas de victimización, y la reacción emocional (el miedo y el deseo de escape) ante la posibilidad de sufrir un delito en una situación real, por ejemplo, cuando se recorre una calle vacía por la noche. Sabemos que un perfil definido por ser mujer, mayor de edad o sin estudios es característico de las personas que dicen sentirse más inseguras, a pesar de no ser quienes generalmente sufren los delitos. Sabemos que los lugares donde ocurren la mayor parte de los delitos no son los mismos en los que la mayoría siente temor. Y sabemos todos de los modelos ecológicos de territorialidad y control social que han dado lugar a las propuestas de prevención del delito a través del diseño urbano (Taylor, 1988; Jacobs, 1961; Newman, 1973), los modelos oportunistas y el carácter profesional del acto delictivo (Brantingham y Brantingham, 1991), la importancia del aspecto físico de abandono (las ventanas rotas de Wilson y Kelling, 1967) y de los lugares con diseños complejos que ofrecen escondites y vías rápidas de escape a los delincuentes (la configuración de misterio analizada por Fischer y Nasar, 1994, o por nosotros mismos en algún trabajo de hace años, Fernández-Ramírez y Corraliza, 1996, por ejemplo). En fin, conclusiones y propuestas que pocos critican y que más o menos todos damos por válidas. No en vano, son el poso de algo más de tres décadas de

Revista Española de Investigación Criminológica

Artículo 6, Número 6 (2008) $\quad$ www.criminología.net

ISSN: $1696-9219$ 
investigación de amplia difusión internacional, que cuenta con un creciente interés también entre los investigadores españoles (Carro, Valera y Vidal, 2005; San Juan, Vergara y Germán, 2005; Vozmediano y San Juan, 2006).

Lo malo de esta situación optimista es que genera un cierto estancamiento en la investigación. Como decía, he tenido que recuperar mis lecturas sobre el tema, y me encuentro con que en los últimos diez años apenas nada ha cambiado, se siguen diciendo las mismas cosas, con el mismo tipo de estudios y resultados similares que ya entonces dábamos por buenos. Claro, ahora hay cierto nivel de detalle que tiene su interés, tal como el estudio de Wang y Taylor (2006) sobre la influencia de la configuración de misterio analizada mediante una simulación de un recorrido a lo largo de distintos callejones urbanos. Sin embargo, la impresión que queda tras la lectura de artículos como el mencionado es que los investigadores se entretienen en un detallismo innecesario, parcial y descontextualizado. En su intento por profundizar, abundan en el detalle y pierden la perspectiva que permite una reflexión molar. (Algo de responsabilidad tienen también los editores y revisores de las revistas, ajustados a un patrón narrativo que remeda el informe experimental sin serlo). Y luego esa sensación de reiteración, de repetición de los mismos temas, los mismos estudios con idénticos planteamientos.

Esto en parte es negativo, pero también me ha dado la pista de que quizá necesitamos pararnos a pensar, a reflexionar sobre esta cantidad de resultados en los que todos estamos aparentemente de acuerdo, pasar de la mera acumulación de estudios empíricos parciales a la crítica y la reflexión teórica. Mi propuesta inicial, como ya he defendido en otras partes, es volver la mirada a los clásicos (Fernández-Ramírez, 2000, 2006). Creo sinceramente que disponemos ahí de un arsenal de ideas, más o menos formalizadas, más o menos sencillas o sutiles, propuestas desde hace ciento cincuenta años, cien años, en los textos de los sociólogos clásicos y en los especialistas clásicos en la teoría social urbana. Y creo que este es el sitio en el que deben enmarcarse los temas de la delincuencia y el miedo al delito, cuya problemática es una más de las muchas caras de la teoría social urbana, de los problemas que derivan de aquella urbanización monstruosa consecuencia de la industrialización del siglo XIX europeo y los comienzos 
del siglo XX norteamericano (el ejemplo de Chicago, paradigma del urbanismo occidental del siglo pasado), hoy generalizada a tantos países de la órbita de la OCDE.

Los epígrafes que estructuran estas páginas recogen seis hipótesis o marcos teóricos posibles que tienen sus raíces en ideas clásicas y encuentran continuidad en autores modernos, en teóricos e ideas recientes, y en investigaciones que continúan realizándose en la actualidad. Es decir, que no estamos realmente fuera de juego, no estamos perdidos, sino que necesitamos detenernos para decidir en dónde estamos y dónde podemos enmarcar nuestro conocimiento. Y estas son, en fin, mis seis hipótesis. Ruego otra vez al amable lector que perdone el riesgo y las licencias conceptuales que me he tomado para construirlas y poner en relación autores e ideas que a veces puedan parecer lejanas. Sólo es la interpretación libre de algunos conceptos y teorías relevantes. El objetivo es sugerir para entender, proponer un punto de partida, o mejor seis, tantos como hipótesis veremos, y nunca un punto de llegada. Empecemos, pues.

\section{La hipótesis del extraño}

Es una idea antigua del sociólogo George Simmel (en Wolf, 1950), cuyos escritos mezclan un lenguaje metafórico sugerente con cierto tinte visionario. La idea del extraño refleja una de las características que han configurado la personalidad del urbanita. Es la idea de que en realidad no nos conocemos, tenemos muchas relaciones diarias, muchas interacciones en muchos espacios de la ciudad, incluso podemos estabilizar una relación, hacerla continua, pero nunca tenemos un conocimiento profundo ni certeza sobre quién es la persona con la que estamos hablando. Si esto lo podemos afirmar de aquellos con los que tenemos un contacto diario (cuántas veces, después de un suceso dramático en el vecindario, los más allegados al homicida, al maltratador o al suicida no afirman lo buena persona que parecía ser, ¡quién iba a pensar que sería capaz de cometer semejante aberración!), si desconozco lo que esconde mi propio vecino, mucho más aquellas personas con las que tenemos un contacto esporádico en la calle. Al cruzar con alguien en la cafetería, en el parque o en cualquier escenario urbano, en cuanto mantenemos ciertos rituales de interacción, la situación nos resulta correcta (el saludo, ceder el paso, los turnos de uso, el comentario intrascendente, la despedida). El otro es un ciudadano más, una persona normal, la interacción funciona, pero desconozco todo lo que hay más allá.

Revista Española de Investigación Criminológica

Artículo 6, Número 6 (2008) $\quad$ www.criminología.net

ISSN: $1696-9219$ 
La contraposición entre lo que la persona deja ver de sí misma y lo que no muestra fue expuesta por Erving Goffman en su conocida obra de 1959 (La presentación del yo en la vida cotidiana). Usando una metáfora teatral, analiza el comportamiento del actor en escenarios sociales en los que representa una actuación ante un público concreto. Cuando yo me presento ante mi audiencia de alumnos y colegas universitarios, adopto ciertas poses que, evidentemente, no son las que utilizo en mi casa cuando me relajo viendo la tele con mi familia. Esta pose es inventada, un truco de actor experimentado. Tengo mis costumbres y mis años de esfuerzo, de aprendizaje del papel, supongo que me sale mejor que años atrás, hasta me siento cómodo porque estoy acostumbrado al papel, incluso a las críticas que conlleva.

Stanley Milgram (1972) realizó una aportación personal a través del concepto de familiar extraño, con el que designa la peculiar relación que establecemos con personas con las que coincidimos a diario en lugares públicos (esperando el metro, por ejemplo), sin que medie interacción alguna (quizá una rápida mirada). Nuestra urbanidad, nuestra capacidad de convivencia ajena a las pautas sociales tradicionales se muestra en el modo en que tácitamente parece que hemos decidido relacionarnos a través de esta ignorancia mutua. A pesar de que cierto tipo de relación existe y del papel que juegan esas otras personas no del todo extrañas para dar continuidad espacial y temporal a nuestra vida diaria.

La cuestión, en fin, es el papel de este desconocimiento mutuo para entender por qué tengo expectativas de ser víctima de un delito en una situación concreta, en la que carezco de claves para descifrar el comportamiento del otro, o las claves son ambiguas y no sé por dónde va a salir la situación, no sé qué va a pasar, no sé quién es esta persona que me mira o camina hacia mí, no sé quién es esta persona con la que ahora me cruzo y que me inspira sentimientos de inseguridad y peligro. El ladrón que roba en la calle juega con cierta apariencia de situación normal, disimula para acercarse a su víctima hasta que es el momento adecuado o queda fuera de la vista de los demás (Felson, 1987). La hipótesis del extraño encaja con esta problemática, insuficientemente estudiada, sobre la expectativa del delito, sobre nuestros estereotipos de la imagen del delincuente y de la situación de peligro. Ayer, por ejemplo, mientas esperaba mi vuelo

Revista Española de Investigación Criminológica 
en el aeropuerto, una persona desconocida me pidió dinero, le miré de reojo y creí reconocer en él muchos años de muchos similares que he visto en mi vida (cierta indumentaria, ciertas posturas del cuerpo al acercarse, cierta entonación familiar en la voz), que desde siempre me han asustado y me siguen asustando todavía. En el escenario del aeropuerto, todavía me cuadraba alguien pidiendo dinero, así que finalmente le di un euro para que me dejara, y de hecho se fue. Pero debo reconocer que, según le miraba la cara, pensé en posibles riesgos y modos de amenaza, y pensé que, en un contexto diferente, habría intentado evitarle e incluso habría acelerado el paso para huir.

Del mismo modo, los especialistas en prejuicio étnico utilizan la hipótesis del extraño para entender el rechazo a la inmigración, la evitación más o menos abierta o sutil que hacemos de su compañía. Y sabemos que una de las estrategias más comunes para la reducción del prejuicio pasa por potenciar el contacto (la interacción) entre niños de diferentes grupos para que mejore el conocimiento mutuo y la aceptación consiguiente del otro (Aronson, 2000; Pettigrew, 1998). Y cuánto habría que hablar aquí sobre el peso de la presencia de inmigrantes en los sentimientos de inseguridad o en la creación de un discurso social de rechazo basado en la atribución de comportamientos delictivos que se hace de los inmigrantes.

\section{La hipótesis psicológica}

Otra idea clásica de George Simmel, expuesta en "La metrópolis y la vida mental" (en Wolf, 1950), la idea del urbanita que ha desarrollado un peculiar estilo de andar por la gran ciudad moderna, ignorante de lo que ocurre alrededor, en una actitud que podríamos pensar fría o desganada (la actitud blasée). Frente al residente de asentamientos tradicionales en comunidades reducidas, está acostumbrado a lidiar diariamente con una multitud de interacciones, situaciones y personas diferentes que demandan su atención; con situaciones concretas en las que hay una variedad incontrolable e imposible de asumir de luces, colores, sonidos, imágenes, letreros, personas que cruzan, todo en el mismo momento o en rápida sucesión. Claro, no se puede estar atento a todo, a la luz, las caras que miran y las que no miran, los múltiples mensajes, y hacer el esfuerzo de pensar sobre todo ello, entenderlo y actuar. Sencillamente, lo que hacemos es ignorar la mayor parte de los mensajes, filtrarlos,

Revista Española de Investigación Criminológica

Artículo 6, Número 6 (2008) $\quad$ www.criminología.net

ISSN: $1696-9219$ 
centrarnos en alguna cuestión de la situación o de las personas que nos parezca relevante por distintas razones, y actuar sólo frente a ella. El resto lo ignoramos. La gran ciudad industrial ha creado una personalidad históricamente nueva, un tipo de personalidad original, que tiene la virtud de resultar adaptativa en un contexto tan complejo, que no es el contexto tradicional de las sociedades humanas, sino el contexto de la sobrecarga de estimulación, como luego lo denominó Milgram (1970).

Nuestra actitud desganada e ignorante de casi todo también tiene sus efectos perversos, como queda ilustrado en el fenómeno de la difusión de la responsabilidad (Latané y Darley, 1970). Es decir, cómo no nos sentimos de ninguna manera responsables de ayudar o de atender a un desconocido que lo requiere porque está viviendo una situación de amenaza (alguien le roba o le agrade en la vía pública), quizá socialmente rechazable (un indigente pidiendo ayuda), quizá sólo alguien ajeno que cae cerca de nosotros víctima de un problema de salud. Sentimos que lo que pase a estas personas no depende de nosotros, es posible que pensemos que no podemos hacer nada o que ya acudirán los servicios públicos dedicados a estas cuestiones (policía, urgencias). Su petición de ayuda, sus rostros se diluyen en todo ese marasmo de cosas que pasan a nuestro alrededor, sobre las que cruzamos sin tener por qué prestarles mayor atención, porque ya lo hará otro o porque no va con nosotros, y basta andar unos metros para que una nueva situación ocupe nuestra atención y nos haga olvidar la anterior.

Este tipo de ideas nos permitiría reflexionar o profundizar en el análisis teórico de algunas cuestiones de interés para la investigación sobre la inseguridad y el miedo al delito. Por ejemplo, sobre la importancia de la expectativa de ayuda cuando nos encontramos en peligro, filtrada por esta ignorancia mutua generalizada, este filtro de no prestar atención a quienes tenemos alrededor. Podríamos entender esa interesante paradoja de que el sentimiento de miedo sea mayor cuando la amenaza ocurre dentro del área residencial más próxima al hogar, en donde se debería esperar precisamente que nuestra sensación de alivio y nuestra expectativa de ser ayudados fuera mayor (Rainwater, 1966; Fernández-Ramírez, 1995). O podríamos, usando el lenguaje del modelo de control ambiental de Lawton (1986; Lawton y Nahemow, 1973), entender que la habilidad para ignorar los problemas alrededor es una suerte de competencia de afrontamiento; es decir, no me asusto porque soy capaz de disimular bien, de hacer

Revista Española de Investigación Criminológica 
como que no pasa nada, que es lo que yo hice ayer con esta persona del aeropuerto de la que antes hablaba, le di el euro que me pedía como si fuera una situación normal, aunque en realidad la situación me resultó desagradable, e incluso sentí cierta inseguridad. Mi reacción de aparente control es un mensaje de tranquilidad para el otro y para mí mismo. La capacidad para desentenderme del conflicto se convierte en una competencia de afrontamiento que pongo en juego cuando quiero resolver una situación de inseguridad.

\section{La hipótesis económica}

Los factores económicos han tenido una gran relevancia en el desarrollo de la teoría urbana, a pesar de que los clásicos fueran criticados en su momento por sugerir cierto determinismo aparentemente burdo. Su importancia se refleja en que este tipo de planteamiento no se ha abandonado con los años, sino que encuentra importantes continuadores en autores de gran relevancia. Una de sus ideas básicas es que los factores económicos condicionan o determinan de algún modo la distribución de los grupos sociales dentro de la ciudad, e incluso la forma de la ciudad. Como describe Lewis Mumford (1961), tenemos ejemplos históricos en el siglo XIX en la configuración de los barrios industriales y sus pésimas condicionas de vida, alejados del control de las instituciones públicas por el interés del gran capital urbano; o el mismo caso de la aparición de la gran ciudad moderna como resultado de las presiones migratorias del campo y del proceso de industrialización que les dio origen. En cuanto a la teoría sobre la forma de la ciudad, Ernest Burgess propone su modelo de anillos concéntricos tratando de representar la distribución de los distritos comerciales, las zonas residenciales de los obreros o las áreas de la industria, es decir, los distintos elementos que entonces formaban el tejido económico de la ciudad (Park, Burgess y McKenzie, 1925).

Claro, no pienso que sea defendible un determinismo economicista rígido, pero sí entiendo que son factores de evidente influencia. Más bien, podríamos orientarlo mediante variables intermedias, como hacía Herbert Gans años después para resolver las críticas, diciendo que la clave no es el mercado, sino que el mercado reacciona ante determinados perfiles socioeconómicos de los grupos sociales en liza (Gans, 1974). La ciudad es el espacio en que los factores y agentes económicos se hacen reales, el

Revista Española de Investigación Criminológica 
escenario del consumo colectivo que da sentido y continuidad a la gran lucha de los intereses económicos (actualizando, con Castells, 1979, el viejo discurso marxista de la lucha de clases). Concentración única de recursos humanos, materiales, informacionales y sobre todo de cadenas de relaciones (contactos, redes sociales, capital social) cambiantes y complejas, ofrecidas a la demanda empresarial como ventaja competitiva. El sistema urbano hace posible el sistema económico, aunque haya efectos en ambas direcciones (Veltz, 2004).

En esta misma línea de argumentación, Harvey (2002) ha analizado las implicaciones del espacio urbanizado como proveedor de infraestructuras y redes de transporte para el desarrollo empresarial, y cómo la acumulación de capital genera un excedente que es invertido en nuevas actuaciones de urbanización (en diversos ciclos históricos ilustrados por la inversión en ferrocarriles, carreteras, canales, edificios de oficinas). En ambos autores, Manuel Castells y David Harvey, se vislumbra así la importancia del sistema de transportes y comunicaciones (la estructura urbana), que se volverá protagonista del nuevo espacio de flujos informacionales de tecnópolis (Castells y Hall, 1994).

Ilustremos la hipótesis económica con el caso actual de la concentración residencial de grupos inmigrantes (Checa y Arjona, 2001). Dado que su capacidad de inversión es muy limitada, sólo determinadas áreas de la ciudad disponen de viviendas a su alcance. Una vez que se empiezan a establecer, se convierten en foco de atracción para los que llegan después, abriendo negocios característicos que ayudan a recrear de algún modo el ambiente social de origen. Es bien visible en la ciudad de Almería, por ejemplo, en donde el centro histórico está renovando su población con inmigrantes magrebíes, con el atractivo añadido de la cercanía al puerto (travesías diarias a localidades norteafricanas). Aunque también la prensa habla desde hace algún tiempo de núcleos residenciales de infravivienda semiocultos en las zonas de invernaderos.

La renovación de la población de un área residencial genera a su vez un desplazamiento de los grupos sociales que tradicionalmente habían ocupado el lugar. La urbanización de nuevas zonas con mayor atractivo residencial (mejores viviendas, mejores servicios, imagen de estatus elevado), reduce la competencia por las zonas viejas, contribuyendo a la caída de los precios, volviéndolas asequibles para los nuevos pobladores de menor

Revista Española de Investigación Criminológica 
poder adquisitivo. Este juego de intereses, reflejado en los cambios del mercado residencial, es evidente en fenómenos como el "efecto donut", en el que las clases medias abandonan las zonas en declive para escapar de los problemas sociales y evitar los impuestos especiales asociados a estas zonas (en países como los Estados Unidos), o los procesos de gentrificación, cuando un grupo social pudiente comienza a interesarse por una zona decaída, contribuyendo a subir los precios con su pujanza, reduciendo de este modo la posibilidad de que los residentes tradicionales opten a continuar en su barrio una vez encarecido (Bounds, 2004). Un ejemplo que roza la perversión está documentado por Foster (2004) en ciudades sudafricanas. La caída del "apartheid" permitió que la población negra optara a una vivienda en zonas residenciales atractivas, decayendo el interés de la elitista población blanca por continuar viviendo en ellas. El desplazamiento ha llevado a crear nuevos barrios de alto "standing", en los que se ha replicado la antigua segregación, ahora legal, mediante el encarecimiento y un tipo de diseño antidelito fuertemente defendible al que algunos llaman "ciudades fortaleza". El valor de la seguridad es uno de los principales argumentos que legitiman esta nueva separación, aunque los factores económicos tienen un papel relevante en el proceso de segregación residencial.

Ningún determinismo ramplón, como se ve, sino un conjunto de fenómenos en los que priman los factores económicos, y que ayudan a entender otra de las caras de los complejos problemas de la criminalidad y la inseguridad.

\section{La hipótesis ecológica}

También podríamos denominarla normativa o del control social. Es la hipótesis más extendida entre nosotros, la más aceptada, quizá la que mejores resultados ha dado, de la que más hablamos normalmente los psicólogos sociales, y me sumo yo también con la investigación realizada bajo la dirección del profesor José Antonio Corraliza, con nuestra modesta aportación sobre el concepto de lugares peligrosos (FernándezRamírez y Corraliza, 1997). El punto de partida son dos conceptos clásicos: la anomia de Emile Durkheim y la contraposición entre comunidad y sociedad de Ferdinand Tönnies (Bettin, 1982). En la comunidad tradicional (la ciudad de dimensiones reducidas o el núcleo rural), el control social está basado en la confianza, en el conocimiento mutuo. No nos puedes atacar porque sabemos quién eres, sabemos

Revista Española de Investigación Criminológica 
quiénes son los tuyos, tu padre, tu familia, de dónde vienes, cuáles son tus debilidades, en fin, lo sabemos todo de ti. En este contexto de proximidad no existe un tipo de delincuencia similar a la que conocemos en la ciudad. Frente a esto, tenemos una sociedad urbana donde el control social no está basado en el conocimiento mutuo, sino que está delegado en controles formales expresamente creados para la seguridad (la policía, el sistema penal). El problema es que la interacción entre los urbanitas es menor, la generación de normas que nosotros mismos nos imponemos durante y como resultado de la interacción para lidiar sobre nuestras relaciones no está presente del mismo modo, la norma social está desaparecida o diluida (no llega a construirse). Puedes atacarnos porque no te sientes obligado hacia nosotros por ninguna norma, dado que ni siquiera nos conocemos y no hemos tenido ocasión de generar normas. En abierta contradicción, la sociedad es anómica, carente de normas, cuando el mismo concepto de sociedad es por definición normativo.

La solución teórica viene de la mano del control social informal (Shaw y McKey, 1942). Aún dentro de la gran urbe, el barrio se convierte en una unidad social intermedia, próxima a la experiencia de convivencia cara a cara de la comunidad tradicional, porque en el barrio todavía no hemos perdido este tipo de relaciones cercanas, nos conocemos, generamos normas, y el control social en este caso no requiere del apoyo de un control formal, al menos no tan presente. Fuera de mi barrio, en una situación potencial de peligro, necesito la presencia del orden público para sentirme seguro; en mi barrio, la mera presencia de las personas, mi conocimiento de los lugares, la proximidad a mi casa, suponen elementos tranquilizadores por sí solos (Fernández-Ramírez y Corraliza, 1998; San Juan, Vergara y Germán, 2005). ¿Cuál es la solución?: crear y afianzar las redes sociales para potenciar los mecanismos del control social informal.

Esta idea, sintetizada de manera muy simple por Jane Jacobs (1961) en la fórmula "ojos que miran a la calle", es la base conceptual del diseño antidelito conocido como espacio defendible,: si hacemos que en todo momento los espacios residenciales estén siendo utilizados o estén siendo observados de manera rutinaria por los residentes y la gente del lugar, crearemos una impresión de vigilancia informal continua y los potenciales delincuentes entenderán que resultan poco adecuados como lugares para el delito,

Revista Española de Investigación Criminológica 
aumentando en consecuencia la seguridad de los residentes. Los originales diseños del arquitecto Oscar Newman (1973), centrados en la disposición de las ventanas y la creación de barreras simbólicas, se han venido multiplicando hasta propuestas como el diseño de recorridos retorcidos para la entrada en la zona residencial o la construcción de barreras reales y sistemas de videovigilancia que convierten los espacios residenciales en "ciudades fortaleza".

El diseño antidelito amplía su base teórica con el discurso de la territorialidad (Taylor, 1987, 1988). En la medida en que el residente se apropia de los espacios residenciales, los personaliza, los utiliza, se sentirá legitimado para defenderlos frente a la invasión de un extraño, poniendo en juego mecanismos de territorialidad. Como sugeríamos hace años (Fernández-Ramírez y Corraliza, 1998), el comportamiento territorial, como fuente de control social informal, ayuda a entender muy diversos fenómenos estrechamente relacionados con la inseguridad, tales como el funcionamiento del diseño antidelito y el espacio defendible; el valor que cobra la red social como un recurso de afrontamiento (la expectativa de ayuda reduce el miedo); la explicación para la teoría de las incivilidades, puesto que el deterioro no asusta, sino que el abandono, el descuido, son signos interpretados como indicios de que no hay orden social, de que no hay norma, no hay control. Y tenemos también buena parte de la explicación de los lugares peligrosos (los hot spots de la inseguridad), que tienden a convertirse en esos pequeños intersticios dentro de nuestro propio área residencial, aquellos lugares en los que no funciona el control social informal, que no forman parte del territorio de los grupos residentes, y acaban siendo ocupados como territorio de grupos que desarrollan actividades marginales (Taylor, 1987).

\section{La hipótesis racionalista}

La perspectiva racionalista está presente desde Max Weber (1972) en buena parte de las ciencias sociales y la psicología, suponiendo que el estudio de las personas requiere analizar el comportamiento intencional, dirigido a meta (Little, 1983; Little, SalmelaAro y Philips, 2006). Sólo podemos anticiparnos y prever lo que hará una persona cuando actúa de manera racional, es decir, cuando se comporta de acuerdo con un plan de actuación molar previsto para conseguir un objetivo deseado. Está en la base del concepto de escenario de conducta, ya que el programa del escenario reúne los planes

Revista Española de Investigación Criminológica

Artículo 6, Número 6 (2008) $\quad$ www.criminología.net

ISSN: $1696-9219$ 
de comportamiento intencionales en el lugar, y es una de las críticas que recibía la ecopsicología de Barker (1968), puesto que el rango de comportamiento potencialmente analizable se limitada a los comportamientos dirigidos a meta (Kaminski, 1983). Está implícita también en el cognitivismo original de la metáfora del ordenador (Neisser, 1976), a partir del cual asumimos que la persona recopila información, la procesa y se comporta en consecuencia, decidiendo líneas de acción que optimizan las relaciones con el ambiente, tal como solemos contar a nuestros alumnos en los temas introductorios de cognición social o de percepción ambiental (Rodríguez Sanabra, 1986).

También las actividades del delito y las cuestiones de la inseguridad forman parte de un cierto racionalismo, tal como se deriva de la idea de transmisión cultural planteada por Clifford Shaw y H. McKey (1942), los criminólogos de la Escuela de Chicago. Un delincuente no es un loco (que serían un porcentaje ínfimo, los que llamaron la atención de Lombroso), sino un profesional de la delincuencia, una persona que tiene una cultura especial bien aprendida, que sabe cómo se comente un robo, cómo funciona el tráfico de drogas o el negocio de la trata de blancas. Y lo ha aprendido conviviendo con sus maestros, ha aprendido cómo se hacen estas cosas, y las sabe hacer bien. Es un profesional con un plan de actuación inteligente, que antes de actuar lo piensa muy bien, es decir, analiza, planifica, actúa y valora (los componentes de la teoría racional de la decisión). Todos conocemos algunas pautas del robo a domicilios. En el delito contra la propiedad, no llega el ladrón y roba una casa, a ver si hay suerte; muy al contrario, los delincuentes están organizados, envían una avanzadilla con uno del grupo que estudia el terreno, señala los mejores objetivos y recopila información sobre las rutinas de los residentes, se planifica el momento y el procedimiento del golpe y se actúa conscientes de las posibilidades de escape (Nelson, 1987). Se trata de un proceso bien pensado, bien organizado, racional, que puede ser descrito de un modo parecido para otro tipo de actos delictivos (contra la persona, delitos sexuales, tráfico de drogas, terroristas, etc.). Los delincuentes forman un grupo aparte, viven en barrios bien conocidos, tienen un argot propio, comparten conocimientos y usos, lugares comunes, elementos que al cabo definen una subcultura propia entre las muchas que habitan la ciudad (Fischer, 1976).

El comportamiento racional de los delincuentes nos lleva a otro tipo de estudios centrados en la idea de oportunidades y riesgos. El delincuente toma sus decisiones

Revista Española de Investigación Criminológica 
porque hace un cálculo de las oportunidades que el escenario le ofrece y los riesgos que le presenta. El delito es viable porque no hay vigilancia, no hay transeúntes accidentales, la vida social está lejos, y al mismo tiempo hay víctimas atractivas (dinero relativamente fácil en el caso de un robo); al contrario, un espacio abierto con tráfico continuo es un lugar rechazado, puesto que podría pasar un coche de policía en cualquier momento y los riesgos aumentan. Y el análisis es más fino incluso de lo que parece en un primer momento, puesto que dentro de un lugar aparentemente arriesgado, en alguna esquina se puede encontrar un punto estratégico suficientemente apartado o escondido, sólo tengo que aprovecharlo para acceder a la vivienda sin ser visto o coger a la persona y arrastrarla dos metros hasta allí en el caso de un robo en la vía pública. En todo ello hay una racionalidad que es previsible, y que permite a Paul y Patricia Brantingham (1991), los criminólogos ambientales, afirmar que, en el análisis del delito, el primer elemento de interés es la dimensión ambiental (el lugar), y predecir la distribución del delito a través de mapas que conjuntan variables de oportunidad y riesgo (distancia desde la vivienda del delincuente, conocimiento de la zona, distribución de víctimas potenciales, etc.). En consecuencia, podemos analizar las actividades rutinarias de los delincuentes y sus víctimas, o mejorar el diseño antidelito incluyendo facetas ambientales que serán interpretadas por el delincuente en términos de oportunidades y riesgos (Kennedy y Forde, 1990).

Y la otra cara de la moneda, la perspectiva racionalista permite entender mejor el comportamiento de la presumible víctima, la persona que siente la inseguridad, porque la evitación de personas o lugares considerados peligrosos es una respuesta que tiene un importante componente racional, además del afectivo. Planificamos la salida de nuestras casas, no atravesamos por cualquier calle, y evitamos o sorteamos de manera inteligente una zona que tenga cierta connotación de inseguridad, o a ciertas horas del día en las que se vuelve insegura. Y también entendemos mejor la importancia de las configuraciones de misterio, que han sido explicadas con argumentos no menos misteriosos. No necesito una hipótesis evolucionista que suponga cómo fue el comportamiento de nuestros antepasados en la filogenia hace algunos millones de años, ni el difícil tránsito entre lo aprendido en un contexto inhóspito y lo transmitido estructuralmente a través de la dotación genética, en una pirueta lamarckiana difícilmente eludible (Appleton, 1975; Kaplan, 1987). Desde mi punto de vista, todo es

Revista Española de Investigación Criminológica

Artículo 6, Número 6 (2008) $\quad$ www.criminología.net

ISSN: 1696-9219 
mucho más sencillo: el misterio, la oscuridad, las sombras, la falta de iluminación, los rincones en una calle, son percibidos como refugios para un potencial delincuente al acecho que aguardara su ocasión (Fisher y Nasar, 1992). Es un lugar adecuado para el delito, según deduzco de mis expectativas racionales sobre la delincuencia. Incluso compartimos una cultura al respecto, como bien saben los directores de películas de terror, que juegan con la tensión de la escena para crear en el espectador la expectativa de un delito inminente. En términos dramatúrgicos (Goffman otra vez) o de psicología ecológica (Barker), los lugares peligrosos se entienden de este modo como el escenario adecuado para el programa que rige la representación del acto delictivo (FernándezRamírez, 1995).

\section{La hipótesis narrativa}

La última hipótesis es la más radical para muchos de nuestros colegas. Sólo plantearla es ya un delicado ejercicio que algunos viven como una crítica excesiva al modo de pensar en el que se han formado y del que muchos nunca saldrán. También es una cuestión de edad, de violencia intergeneracional. He escuchado recientemente a un grupo de criminólogos de gran reputación que exponían y comentaban unos gráficos sobre la incidencia y prevalencia de diferentes delitos. El discurso de la ciencia convencional se traslucía en ciertas partes, en ciertas expresiones que forman parte del ritualizado lenguaje científico. Por ejemplo, el modo en que daban por buenos los datos presentados bajo una supuesta objetividad que los legitima. Nuestros compañeros nos ofrecen unos datos que parecen quedar demostrados por sí mismos, pero que son utilizados estratégicamente para justificar un discurso que más bien parecía el discurso que querían defender. No critico el valor de sus datos (aunque podría, dado que los supuestos epistemológicos y metodológicos de partida son criticados, y bien, por ejemplo por Ibáñez, 1990, 2002, o Guba y Lincoln, 1989), ni el esfuerzo o el mérito de sus investigaciones. Lo que me resultaba criticable era el salto realizado desde los gráficos al discurso. Sobre cada uno de los datos comentados, y en muchos que seguramente pasaban desapercibidos al auditorio, yo me hacía preguntas incesantemente pensando "ipero ese dato no es tan correcto!, depende de que consideremos otros datos que faltan, ¿dónde están?”, “isi no conozco la evolución de ese índice!, nos ofrecen datos estáticos, pero cómo interpretar el número de delitos si no conocemos el tamaño de la población, cómo valorar el número de delitos si no conocemos los tipos de delitos

Revista Española de Investigación Criminológica

Artículo 6, Número 6 (2008) $\quad$ www.criminología.net

ISSN: $1696-9219$ 
y con qué criterios se establece la tipología considerada". Pero nuestros colegas dan el salto con facilidad asumiendo que el dato objetivo, duro, demuestra por sí solo un discurso que ha sido construido con anterioridad. Creo que buena parte del problema está en la interpretación y en la comprensible necesidad que tenemos de generar discursos coherentes. Y conste que no me parece mal: el discurso resultante estaba bien argumentado y era coherente, claro y defendible.

Estamos pues en la crítica de los discursos. Cuidado, no criticamos el lenguaje, sino que elaboremos nuestros brillantes discursos científicos ignorando la capacidad reificadora que tienen. Es nuestra querida posmodernidad, nuestra actualidad, el espíritu de nuestro tiempo. Somos todos posmodernidad porque es el momento histórico que nos toca, queramos o no, incluso los que pretenden mantenerse en la modernidad pasada resultan posmodernos en su intento de quedarse atrás (argumentos posmodernos para defender un modernismo rebasado). Situados en un cruce de caminos donde los discursos, las narrativas, los textos trasladan, crean y legitiman realidades en consonancia con las fuentes del poder (Lyotard, 1989), académico en este caso, nuestros compañeros de avanzada edad dictando cátedra investidos de la dignidad y el prestigio de los años, la experiencia, los contactos. Poder, discurso y legitimación enmarcan una realidad fragmentada que ha dejado de serlo, donde cada nuevo mito (la religión, la ciencia, la lucha de clases, la globalización) se impone sobre el anterior, lo sustituye tajantemente legitimando nuevas prácticas sociales, nuevas normas y estructuras de poder (Foucault, 1976). Mucho de relevo generacional y disputa por el poder académico, ya digo.

Los temas del posmodernismo son variados y apenas si tienen como punto en común la cuestión de la narratividad (la hermenéutica) y la actitud de rechazo hacia los grandes valores y prácticas características de la ciencia modernista (crítica al valor ilustrado del progreso, a la deificación de la ciencia como verdad absoluta, crítica a todos los conceptos y marcos con pretensión de absoluto, crítica al método científico clásico y al operacionalismo como herramientas para descubrir la verdad, etc.).

Hay mucho en algunas propuestas posmodernistas que tienen que ver con la perspectiva psicosocial que defienden nuestros maestros (Blanco, 1996; Gergen, 1985), es decir, que no hay realidades puras, y menos las realidades sociales, todas ellas son fruto de

Revista Española de Investigación Criminológica 
una construcción, de un proceso de redefinición continuo protagonizado por los grupos sociales interesados en el problema de análisis. Así, la evaluación de cuarta generación de Egon Guba e Yvone Lincoln (1989), definida como construccionista y naturalista, que condiciona los planteamientos del proceso de intervención social y evaluación de programas a un proceso de negociación a múltiples bandas en el que el evaluador asume el papel de negociador que pone los intereses grupales sobre la mesa y ayuda a buscar consensos. O el análisis del poder y el discurso de Michel Foucault (1976), que muestra el valor de los discursos públicos como mecanismos de legitimación del estatus de los grupos situados en posiciones de poder, discursos que son asumidos (interiorizados) por el individuo condicionando su forma de entender y de entenderse en el mundo.

Los vínculos entre poder y discurso se nos hacen evidentes a diario en los periódicos, cuando cada partido político o cada diario cuenta la noticia afirmando determinadas cosas de determinada manera (escoge la información y la estructura para mantener una posición de interés sospechosamente predefinida, igual que hacían nuestros compañeros de ciencia, igual que estoy haciendo yo ahora al preparar este capítulo), afirmaciones que son reinterpretadas y reenmarcadas por sus adversarios para ser dichas de otro modo definiendo discursos e intereses alternativos, afirmaciones que son también interiorizadas, creídas y defendidas a pies juntillas por los seguidores de cada grupo. Más aún, nuestro problema surge ante la duda más que razonable de que nosotros mismos, los investigadores, estemos instrumentalizando unos "datos" cuya objetividad es cuestionable, a favor de tesis previas cuyos supuestos básicos no pueden ser cuestionados o no se hacen explícitos. ¡Que además nos creamos que el discurso resultante sea la verdad! ¡Yen pleno siglo XXI!

En fin, cuando pensamos en la delincuencia y la inseguridad, también nuestros argumentos y nuestros datos forman parte de discursos sociales que responden a presiones de distintos grupos que tienen un componente político, muchas veces de manera sutil, mas nunca inocente, dados los intereses en juego. Así, las feministas critican la investigación que afirma que las mujeres son más inseguras, porque esto refuerza un discurso que relega a las mujeres a un papel pasivo y dependiente (Koskela, 1997; Sherman, 1998). Nos preguntamos qué pasa con las mujeres en la ciudad y eludimos la pregunta sobre los hombres. Quizá no es que ellas sean más inseguras, sino

Revista Española de Investigación Criminológica

Artículo 6, Número 6 (2008) $\quad$ www.criminología.net

ISSN: $1696-9219$ 
que ellos se muestran en exceso seguros, ¿por qué? Y centramos el discurso en la mujer víctima porque es coherente con otro discurso social ya establecido y no completamente criticado, el de la indefensión de la mujer que necesita un protección especial. Sólo cuando analizamos los discursos con un ánimo crítico, los cuestionamos y les damos la vuelta, somos capaces de darnos cuenta del sutil juego de los intereses y de la construcción interesada de la verdad. Otro ejemplo, ¿Qué es un delito?, ¿qué tipos de delito hay?, ¿debemos considerar delincuente a uno de estos gorrillas, como dicen en el sur, estos personajes que cobran un canon por dejarnos estacionar nuestro coche en un aparcamiento público?, ¿no es un sutil chantaje? ¿Y la violencia doméstica es un delito, aunque hace veinte años no se hablara de ella como hoy se hace, quizá porque entonces se consentía, tenía una legitimidad, una explicación ¿por qué es un delito hoy y entonces no? ¿Por qué no puedo cuestionar que ciertos delitos, como la violencia doméstica o el terrorismo, sean considerados como tales sin recibir acusaciones de maltratador o cooperador? Quizá porque existen discursos nuevos legitimados desde ciertos grupos con influencia y aceptados públicamente, y la crítica reduce su legitimidad tanto como da argumentos para justificar la posición contraria, así que debemos concluir que para entender los problemas de la delincuencia y la inseguridad debemos asumir que estamos hablando dentro de discursos que tienen un poder para definir la realidad y para legitimar perspectivas de interés.

\section{Conclusión}

Pido disculpas si la exposición ha podido parecer incompleta en alguno de sus apartados. A mi vuelta a los estudios urbanos se suman mis actuales inquietudes posmodernas y el hecho de que el texto sea una mezcla de lenguaje hablado y escrito como fruto de la corrección y ampliación de una ponencia impartida ante un auditorio. No ocultaré, sin embargo, que me he sentido cómodo al poder exponer fragmentos de ideas sin sentir la obligación de alcanzar una coherencia completa, un saber definitivo sobre la cuestión urbana y los fenómenos de la inseguridad que, ya he defendido en ocasiones anteriores (Fernández-Ramírez, 2000, 2006), más bien se caracterizan por la multitextualidad, la pluralidad de fenómenos, objetos, situaciones y discursos que convergen en el interés por hablar de ciudades y de teoría social urbana. 
Queda para un posterior esfuerzo la tarea de completar cada hipótesis, revisar, reinterpretar y sintetizar con más detalle el pensamiento de cada uno de los grandes autores que han ido apareciendo a lo largo de este capítulo. Necesitamos pensar más y contextualizar mejor los resultados empíricos y las propuestas teóricas. Hay mucho trabajo por hacer, mucha información, muchos temas que estudiar. Creo, como propuse al comienzo, que deberíamos contextualizarlos dentro de la teoría social urbana, hablar de la inseguridad y del delito hablando de las cuestiones urbanas, que es el espacio intelectual en que encuentran sentido teórico, metodológico y experiencial.

Entiendo que las bases teóricas más aceptadas en la investigación actual sobre delincuencia y miedo al delito se engloban bajo la hipótesis ecológica (anomia, control social, territorialidad, lugares peligrosos). No es la única, como hemos visto suficientemente, pero sí la que más se utiliza como fondo teórico de la investigación que ahora se hace y la que mayor potencial tiene para asumir propuestas planteadas desde otras hipótesis. Además, aquí deseo romper una lanza para introducir la hipótesis narrativa y los planteamientos posmodernos en la teoría urbana que se desarrolla en nuestro país, donde apenas nuestros colegas de las universidades catalanas son la honrosa avanzadilla (Vivas, Mora, Vidal y otros, 2005). Camino quizá de un urbanismo posmodernista que rechaza las pautas racionalistas de la megaconstrucción, la monumentalidad y el automóvil como base para el diseño urbano, a favor de espacios fragmentados, próximos a una dimensión humana, decorativos, que priman y recuperan identidades locales (Dear y Flusty, 2002; Relph, 1987). La sustitución quizá del urbanismo de Chicago, que ha servido como modelo y como espacio de discusión para varias generaciones de urbanistas y teóricos sociales, asumiendo, como sugiere Dear (2000), el caos y el multicentrismo fragmentado de Los Ángeles como modelo de urbanismo para el siglo XXI. 


\section{Referencias}

Appleton, J. (1975). The experience of place. Londres: Wiley.

Aronson, E. (2000). El animal social (8 ${ }^{\mathrm{a}} \mathrm{ed}$.). Madrid: Alianza.

Barker, R.G. (1968). Ecological psychology: Concepts and methods for studying the environment of human behavior. Stanford, CA: Stanford University Press.

Bettin, G. (1982). Los sociólogos de la ciudad. Barcelona: Gustavo Gili.

Blanco, A. (1996). Vygotski, Lewin y Mead: los fundamentos clásicos de la Psicología social. En D. Paéz y A. Blanco (Eds.), La teoría sociocultural y la Psicología social actual (pp. 27-62). Madrid: Fundación Infancia y Aprendizaje.

Bounds, M. (2004). Urban social theory. City, self, and society. Victoria, Australia: Oxford University Press.

Brantingham, P.J. y Brantingham, P.L. (1991). Environmental criminology. (2a ed.). Beverly Hills, CA: Sage.

Carro, D., Valera, S. y Vidal, T. (2005). Inseguridad percibida en el espacio público: variables personales, sociales y ambientales en un estudio de un barrio de Barcelona. En R. García-Mira, A. Fernández, M.D. Losada y M. Golugoff (Comps.), Psicología Ambiental, Comunitaria y de la Educación (pp. 65-73). Madrid: Biblioteca Nueva.

Castells, M. (1979). La cuestión urbana. Madrid: Siglo XXI.

Castells, M. y Hall, P. (1994). Las tecnópolis del mundo. La formación de los complejos industriales del siglo XXI. Madrid: Alianza.

Checa, J.C. y Arjona, A.. (2001). El Ejido: de erial urbano a ciudad-cortijo. En F. Checa (dir.), El Ejido: la ciudad-cortijo. Claves socioeconómicas del conflicto étnico (pp. 127-170). Barcelona, Icaria.

Dear, M.J. (2000). The postmodern urban condition. Oxford, UK: Blackwell.

Dear, M.J. y Flusty, S. (2002). Postmodern urbanism. En M.J. Dear y S. Flusty (Eds.), The spaces of postmodernity: A reader in human geography (pp. 216-234). Oxford, UK: Blackwell.

Felson, M. (1987). Routine activities and crime prevention in the developing metropolis. Criminology, 25(4), 911-931. 
Fernández-Ramírez, B. (1995). Lugares peligrosos. Psicología ambiental y miedo al delito. Madrid: Universidad Autónoma de Madrid.

Fernández-Ramírez, B. (2000). El medio urbano. En J.I.Aragonés y M. Amérigo (Coords.), Psicología Ambiental (pp. 259-280.) Madrid: Pirámide.

Fernández-Ramírez, B. (2006). Un breve apunte para enmarcar la psicología ambiental de la ciudad. En J.A. Corraliza, J. Berenguer y R. Martín (Eds.), Medio ambiente, bienestar humano y responsabilidad ecológica (pp. 453-459). Madrid: Resma y Fundación General de la UAM.

Fernández-Ramírez, B. y Corraliza, J.A. (1996). Aspectos físicos y sociales en los lugares peligrosos: Miedo al delito en un espacio institucional. Revista de Psicología Social, 11(2), 219-234.

Fernández-Ramírez, B. y Corraliza, J. A. (1997). Hacia una tipología de lugares peligrosos, en relación con el miedo al delito. Intervención Psicosocial, 6(2), 237-248.

Fernández-Ramírez, B. y Corraliza, J.A. (1998). Generalidad y especificidad en la explicación del miedo al delito. Apuntes de Psicología, 16(12): 173-186.

Fischer, C. (1976). The urban experience. Nueva York: Harcourt Brace Jovanovitch.

Fisher, B. y Nasar, J. (1992). Fear of crime in relation to three exterior site features, Environment and Behavior, 24(1) 35-65.

Foster, N. (2004). Crime, fear and the gate. Prospects of resurgence in South African cities. Presentado en Leverhulme International Symposium 2004: The resurgent city. Londres: London School of Economics, 19-21 de Abril. Recuperado el 25 de Mayo de 2007 desde la página de la London School of Economics and Political Science:

http://www.lse.ac.uk/collections/resurgentCity/Papers/NinaFoster.pdf

Foucault, M. (1976). Vigilar y castigar. México: Siglo XXI.

Gans, H. (1974). Urbanism and suburbanism as ways of life. En R. Pahl (Ed.), Readings in urban sociology (pp. 95-118). Oxford, UK: Pergamon.

Gergen, K.J. (1985). The social constructionist movement in social knowledge. American Psychologist, 40(3), 266-275.

Goffman, E. (1959). The presentation of self in everyday life. Londres: Penguin. (Trad. española en La presentación de la persona en la vida cotidiana, Buenos Aires, Amorrortu, 1993) 
Guba, E. G. y Lincoln, Y. S. (1989). Fourth generation evaluation. Newbury Park, CA: Sage.

Hale, C. (1996). Fear of crime: a review of the literature. International Review of Victimology, 4(2), 79-150.

Harvey, D. (2002). The urban process under capitalism: a framework for analysis. En G. Bridge y S. Watson (Eds.), The Blackwell City Reader (pp. 116-124). Oxford, UK: Blackwell.

Ibáñez, T. (1990). Aproximaciones a la psicología social. Barcelona: Sendai.

Ibáñez, T. (2002). Municiones para disidentes. Realidad - Verdad - Política. Barcelona: Gedisa.

Jacobs, J. (1961). The death and life of great American cities. Nueva York: Vintage.

Kaminski, G. (1983). The enigma of ecological psychology. Journal of Environmental Psychology, 3, 85-94.

Kaplan, S. (1987). Aesthetic, affect and cognition. Environmental preference from an evolutionary perpective. Environment and Behavior, 19(1), 3-31

Kennedy,L.W. y Forde, D.R. (1990). Routine activities and crime: An analysis of victimization in Canada. Criminology, 28(1), 137-151.

Koskela, H. (1997). Bold walk and breakings: women's spatial confidence versus fear of violence. Gender, Place and Culture, 4(3), 301-319.

Latané, B. y Darley, J. M. (1970). The unresponsive bystander: Why doesn't he help? Engewood Cliff, NJ: Prentice-Hall.

Lawton, M.P. (1986). Environment and aging ( $2^{\text {nd }}$ ed.). Albany, NY: Center for the Stufy of Aging.

Lawton, M.P. y Nahemow, L. (1973). Ecology and the aging process. En C. Eisdorfer y M.P. Lawton (Eds.), Psychology of adult development and aging (pp. 619-674). Washington: American Psychological Association.

Little (1987) es Little, B.R. (1983). Personal projects: A rationale and method for investigation. Environment and Behavior, 15(3), 273-309.

Little, B.R., Salmela-Aro, K. y Philips, S.D., eds. (2006). Personal Project pursuit. Goals, action, and human flourishing. Mahwah, NJ: Erlbaum.

Lyotard, J.F. (1989). La condición postmoderna. Informe sobre el saber. Madrid: Cátedra.

Milgram, S. (1970). The experience of living in cities. Science, 167, 1461-1468.

Revista Española de Investigación Criminológica

Artículo 6, Número 6 (2008) $\quad$ www.criminología.net

ISSN: $1696-9219$ 
Mumford, L. (1961). The city in History: Its origins, its transformations, and its prospects. Nueva York: Harcourt Brace Jovanovitch.

Neisser (1976). Cognition and Reality. San Francisco, CA: Freeman.

Newman, O. (1972). Defensible space. Crime prevention through urban design. Nueva York: Macmillan

Park, R.E., Burgess, E.W. y McKenzie, R.D. (1925). The City. Chicago, IL: University of Chicago Press.

Pettigrew, T. (1998). Intergroup contact theory. Annual Review of Psychology, 49, 6585.

Rainwater, L. (1966). Fear and house-as-haven in the lower class. Journal of the American

Institute of Planners, 32, 23-31.

Relph, E.C. (1987). The modern urban landscape. Baltimore, MD: The Johns Hopkins University Press.

Rodríguez Sanabra, F. (1986). Percepción ambiental. En F. Jiménez Burillo y J.I. Aragonés (Eds.), introducción a la Psicología ambiental (pp. 21-34). Madrid: Alianza.

San Juan, C., Vergara, A. y Germán, I. (2005). Propiedades psicométricas de un cuestionario para la evaluación de la calidad de vida urbana y el miedo al delito. Revista Española de Investigación Criminológica, 3, 1-13.

Shaw, C. y McKay, H. (1942). Juvenile Delinquency and Urban Areas. Chicago: University of Chicago Press.

Sherman, L.W. (1998). Policing for crime prevention. En L.W. Sherman, D. Gottfredson, D. MacKenzie, D., Eck, J., Reuter, P., Bushway, S. (Eds), Preventing Crime: What Works, What Doesn't, What's Promising. Department of Criminology and Criminal Justice, University of Maryland. Recuperado el 6 de mayo de la página del NCJRS :

http://www.ncjrs.gov/works/index.htm

Taylor, R.B. (1987). Toward an environmental psychology of disorder: delinquency, crime, and fear of crime. En D. Stokols e I. Altman (Eds.), Handbook of environmental psychology (951-986). Nueva York: Wiley. 
Taylor, R.B. (1988). Human Territorial Functioning: An Empirical Evolutionary Perspective on Individual and Small Group Territorial Cognitions, Behaviors, and Consequences. Nueva York: Cambridge University Press.

Veltz, P. (2004). The rationale for a resurgence in the major cities of advanced economies. Presentado en Leverhulme International Symposium 2004: The resurgent city. Londres: London School of Economics, 19-21 de Abril. Recuperado el 25 de Mayo de 2007 desde la página de la London School of Economics and Political Science:

http://www.lse.ac.uk/collections/resurgentCity/Papers/Opening\%20plenary/pierreveltz. pdf

Vivas, P., Mora, M., Vidal, T. y otros (2005). Ventanas en la ciudad. Observaciones sobre las urbes contemporáneas. Barcelona: UOC.

Vozmediano, L. y San Juan, C. (2006). Empleo de Sistemas de Información Geográfica en el estudio del miedo al delito. Revista Española de Investigación Criminológica, 4, 1-11.

http://www.criminologia.net/contenidos/revistaContenido/4/revista4-2006_Articulo.pdf

Wang, K. y Taylor, R.B. (2006). Simulated walks through dangerous alleys: Impacts of features and progress on fear. Journal of Environmental Psychology, 26, 269283.

Weber, M. (1972). Ensayos de sociología contemporánea. Barcelona: Martínez Roca.

Wilson, J.Q. y Kelling, G. (1982). Broken windows: The police and neighborhood safety. The Atlantic Monthly, 127, 29-38.

Wolff, K. (1950). The Sociology of Georg Simmel. Nueva York: Free Press. 